\title{
TITLE:
}

\section{WKB approximation for multichannel barrier penetrability}

$\operatorname{AUTHOR}(\mathrm{S})$ :

Hagino, K; Balantekin, AB

\section{CITATION:}

Hagino, K ... [et al]. WKB approximation for multichannel barrier penetrability. PHYSICAL REVIEW A 2004, 70(3): 032106.

ISSUE DATE:

2004-09

URL:

http://hdl.handle.net/2433/50487

RIGHT:

Copyright 2004 American Physical Society 
PHYSICAL REVIEW A 70, 032106 (2004)

\title{
WKB approximation for multichannel barrier penetrability
}

\author{
K. Hagino ${ }^{1,2}$ and A. B. Balantekin ${ }^{3}$ \\ ${ }^{1}$ Yukawa Institute for Theoretical Physics, Kyoto University, Kyoto 606-8502, Japan \\ ${ }^{2}$ Department of Physics, Tohoku University, Sendai 980-8578, Japan \\ ${ }^{3}$ Department of Physics, University of Wisconsin, Madison, Wisconsin 53706, USA
}

(Received 30 April 2004; published 21 September 2004)

\begin{abstract}
Using the method of the local transmission matrix, we generalize the well-known WKB formula for barrier penetrability to multichannel systems. We compare the WKB penetrability with a solution of the coupledchannels equations, and show that the WKB formula works well at energies well below the lowest adiabatic barrier. We also discuss the eigenchannel approach to a multichannel tunneling, which may improve the performance of the WKB formula near and above the barrier.
\end{abstract}

DOI: 10.1103/PhysRevA.70.032106

\section{INTRODUCTION}

The coupled-channel approach has been a standard method in describing atomic, molecular, and nuclear reactions involving internal degrees of freedom [1-5]. In nuclear physics, for instance, the coupled-channel method has been successfully applied to heavy-ion fusion reactions at energies around the Coulomb barrier in order to discuss the effect of couplings between the relative motion of the colliding nuclei and inelastic excitations in the target nucleus [1-3]. At these energies, the fusion reaction takes place by quantum tunneling, and the coupled-channel calculations account well for the enhancement of the barrier penetrability due to the channel couplings.

A difficulty in the coupled-channel calculations, however, is that it is sometimes not so easy to obtain a numerically stable solution with a controlled accuracy. This is particularly the case in the presence of closed channels and/or when the coupling strength is strong in the classically forbidden region. Several methods have been proposed in order to stabilize the numerical solution [4,6-8].

In this paper, instead of directly integrating the coupledchannel equations with the stabilization techniques, we solve them using the WKB approximation [9]. To this end, we employ the method of the local transmission matrix, which was originally developed by Brenig and Russ in order to stabilize numerical solutions of the coupled-channel equations [4]. We solve the equation for the local transmission matrix under the semiclassical assumption, and generalize the well-known WKB formula for barrier penetrability for a single channel to coupled-channel systems. Since the penetrability is expressed in a compact form, the resultant WKB formula is entirely free from the problem of numerical instability. Moreover, the WKB method can be easily applied to systems with a large number of degrees of freedom, while obtaining a direct solution of the coupled-channel equations can be computationally demanding. Also, the WKB method is useful in gaining a physical intuition for the dynamics of multichannel tunneling.

The paper is organized as follows. In Sec. II, we set up the coupled-channel equations and introduce the local transmission matrix. We derive the semiclassical expression for the local transmission matrix, and obtain the WKB formula for
PACS number(s): 03.65.Xp, 03.65.Sq, 24.10.Eq, 25.70.Jj

multichannel penetrability. We apply the WKB formula to a three-channel problem, and compare the penetrability with the numerical solutions of the coupled-channel equations. In Sec. III we discuss the penetrability at energies near and above the barrier height. The WKB formula which we derive works when the multiple reflection of the classical path under the barrier can be neglected, that is, at energies well below the barrier. We show that the eigenchannel approach can provide good prescriptions at higher energies, where the primitive WKB formula breaks down. We summarize the paper in Sec. IV.

\section{MULTICHANNEL WKB FORMULA}

Our aim in this paper is to derive the WKB formula for penetrability for a one-dimensional potential barrier in the presence of channel couplings. We consider the following coupled-channel equations:

$$
-\frac{\hbar^{2}}{2 m} \frac{d^{2}}{d x^{2}} u_{n n_{0}}(x)+\sum_{m}\left[V_{n m}(x)+\left(\epsilon_{m}-E\right) \delta_{n, m}\right] u_{m n_{0}}(x)=0 \text {. }
$$

Here, $m$ is the mass of a particle, $\epsilon_{n}$ is the excitation energy for the $n$th channel, and $E$ is the total energy of the system. $u_{n n_{0}}(x)$ is the wave function matrix, where $n$ refers to the channel while $n_{0}$ specifies the incident channel. Notice that we express the wave functions in a matrix form by combining $N$ linearly independent solutions of the coupled-channel equations, $N$ being the dimension of the coupled equations. For the situation where the particle is incident on the barrier from the right hand side, the boundary conditions for $u_{n n_{0}}(x)$ are given by

$$
\begin{aligned}
u_{n n_{0}}(x) & \rightarrow T_{n n_{0}} e^{-i k_{n} x} \quad(x \rightarrow-\infty), \\
& \rightarrow \delta_{n n_{0}} e^{-i k_{n} x}+R_{n n_{0}} e^{i k_{n} x} \quad(x \rightarrow \infty),
\end{aligned}
$$

where $k_{n}=\sqrt{2 m\left(E-\epsilon_{n}\right) / \hbar^{2}}$ is the wave number for the $n$th channel. The inclusive penetrability is then obtained as 


$$
P=\sum_{n} \frac{k_{n}}{k_{n_{0}}}\left|T_{n n_{0}}\right|^{2}
$$

Let us now introduce the local transmission matrix defined by [4]

$$
\boldsymbol{\tau}(x) \equiv \frac{1}{2}\left[i \mathbf{q}(x)^{-1} \mathbf{u}^{\prime}(x)+\mathbf{u}(x)\right]
$$

where $\mathbf{q}(x)=\left\{2 m[E-\mathbf{W}(x)] / \hbar^{2}\right\}^{1 / 2}$ with $W_{n m}(x)=V_{n m}(x)$ $+\epsilon_{n} \delta_{n, m}$. From Eqs. (2) and (3), the asymptotic form of $\boldsymbol{\tau}(x)$ reads

$$
\begin{aligned}
\tau_{n m}(x) & \rightarrow T_{n m} e^{-i k_{n} x} \quad(x \rightarrow-\infty), \\
& \rightarrow \delta_{n, m} e^{-i k_{n} x} \quad(x \rightarrow \infty) .
\end{aligned}
$$

Here, we have used the fact that $q_{n m}(x) \rightarrow k_{n} \delta_{n, m}$ as $x \rightarrow \pm \infty$. It is easy to show that the local transmission matrix $\boldsymbol{\tau}(x)$ obeys the equation [4]

$$
\boldsymbol{\tau}^{\prime}(x)=-\frac{1}{2} \mathbf{q}^{-1}(x) \mathbf{q}^{\prime}(x)[1+\boldsymbol{\rho}(x)] \boldsymbol{\tau}(x)-i \mathbf{q}(x) \boldsymbol{\tau}(x),
$$

where

$$
\boldsymbol{\rho}(x) \equiv\left[i \mathbf{q}^{-1}(x) \mathbf{u}^{\prime}(x)-\mathbf{u}(x)\right] \cdot\left[i \mathbf{q}^{-1}(x) \mathbf{u}^{\prime}(x)+\mathbf{u}(x)\right]^{-1}
$$

is the local reflection matrix [4].

The WKB approximation may be obtained by neglecting $\boldsymbol{\rho}(x)$ in Eq. (8) $[10,11]$, that is,

$$
\boldsymbol{\tau}^{\prime}(x)=-\frac{1}{2} \mathbf{q}^{-1}(x) \mathbf{q}^{\prime}(x) \boldsymbol{\tau}(x)-i \mathbf{q}(x) \boldsymbol{\tau}(x)
$$

A similar equation has been derived by Van Dijk and Razavy $[10,11]$, but by using the method of variable reflection amplitude (see also Ref. [12]). Notice that the asymptotic form of the local reflection matrix $\boldsymbol{\rho}(x)$ is [4]

$$
\begin{aligned}
\rho_{n m}(x) & \rightarrow 0 \quad(x \rightarrow-\infty), \\
& \rightarrow-R_{n m} e^{2 i k_{n} x} \quad(x \rightarrow \infty) .
\end{aligned}
$$

Neglecting $\boldsymbol{\rho}(x)$ in Eq. (8) is thus equivalent to ignoring the reflection, which is reasonable in the semiclassical limit. This, in fact, corresponds to the lowest order of the Bremmer expansion [13-17], where the WKB formula is obtained by approximating a smooth potential with a series of sharp potential steps. ${ }^{1}$

For a single-channel problem, Eq. (10) can be easily integrated to yield

\footnotetext{
${ }^{1}$ The local reflection matrix $\boldsymbol{\rho}(x)$ satisfies the equation $\boldsymbol{\rho}^{\prime}=i(\mathbf{q} \boldsymbol{\rho}$ $+\boldsymbol{\rho q})-(1-\boldsymbol{\rho}) \mathbf{q}^{-1} \mathbf{q}^{\prime}(1+\boldsymbol{\rho}) / 2$ [4]. One may solve this equation perturbatively assuming that $\boldsymbol{\rho}(x)$ remains small and finding the correction to Eq. (10).
}

$$
\tau(x)=\sqrt{\frac{q(\infty)}{q(x)}} \exp \left(-i \int^{x} q\left(x^{\prime}\right) d x^{\prime}\right) .
$$

For a coupled-channel problem, however, care must be taken in the integration, since $\mathbf{q}(x)$ and $\mathbf{q}^{\prime}(x)$ do not commute to each other in general. We attempt to solve Eq. (10) by discretizing the coordinate with a mesh spacing of $\Delta x$. Replacing the derivative term by a simple point difference formula, we obtain

$$
\begin{aligned}
\boldsymbol{\tau}\left(x_{n-1}\right) & \sim \boldsymbol{\tau}\left(x_{n}\right)+\Delta x\left[i \mathbf{q}\left(x_{n}\right) \boldsymbol{\tau}\left(x_{n}\right)+\frac{1}{2} \mathbf{q}^{-1}\left(x_{n}\right) \mathbf{q}^{\prime}\left(x_{n}\right) \boldsymbol{\tau}\left(x_{n}\right)\right] \\
& \sim\left[1+\frac{\Delta x}{2} \mathbf{q}^{-1}\left(x_{n}\right) \mathbf{q}^{\prime}\left(x_{n}\right)\right]\left[1+i \mathbf{q}\left(x_{n}\right) \Delta x\right] \boldsymbol{\tau}\left(x_{n}\right) \\
& \sim\left[1-\Delta x \mathbf{q}^{-1}\left(x_{n}\right) \mathbf{q}^{\prime}\left(x_{n}\right)\right]^{-1 / 2} e^{i \mathbf{q}\left(x_{n}\right) \Delta x} \boldsymbol{\tau}\left(x_{n}\right)
\end{aligned}
$$

to the lowest order of $\Delta x$. Using $\mathbf{q}\left(x_{n-1}\right) \sim \mathbf{q}\left(x_{n}\right)[1$ $\left.-\Delta x q^{-1}\left(x_{n}\right) \mathbf{q}^{\prime}\left(x_{n}\right)\right]$, the first factor in Eq. (16) is transformed to be

$$
\left[1-\Delta x \mathbf{q}^{-1}\left(x_{n}\right) \mathbf{q}^{\prime}\left(x_{n}\right)\right]^{-1 / 2} \sim \frac{1}{\sqrt{\mathbf{q}^{-1}\left(x_{n}\right) \mathbf{q}\left(x_{n-1}\right)}} \frac{1}{\sqrt{\mathbf{q}\left(x_{n}\right)}} \sqrt{\mathbf{q}\left(x_{n}\right)} .
$$

Approximating $\quad\left[\mathbf{q}^{-1}\left(x_{n}\right) \mathbf{q}\left(x_{n-1}\right)\right]^{-1 / 2}\left[\mathbf{q}\left(x_{n}\right)\right]^{-1 / 2}$ $\sim\left[\mathbf{q}\left(x_{n}\right) \mathbf{q}^{-1}\left(x_{n}\right) \mathbf{q}\left(x_{n-1}\right)\right]^{-1 / 2}=1 / \sqrt{\mathbf{q}\left(x_{n-1}\right)}$, we finally obtain

$$
\boldsymbol{\tau}\left(x_{n-1}\right)=\frac{1}{\sqrt{\mathbf{q}\left(x_{n-1}\right)}} e^{i \mathbf{q}\left(x_{n}\right) \Delta x} \sqrt{\mathbf{q}\left(x_{n}\right)} \boldsymbol{\tau}\left(x_{n}\right) .
$$

Iterating this equation backward from $x=\infty$, we obtain

$$
\boldsymbol{\tau}(-\infty)=\frac{1}{\sqrt{\mathbf{q}(-\infty)}}\left(\prod_{i} e^{i \mathbf{q}\left(x_{i}\right) \Delta x}\right) \sqrt{\mathbf{q}(\infty)} .
$$

Substituting this expression into Eq. (4) together with Eq. (6), the WKB approximation to the multichannel penetrability reads

$$
P=\sum_{n}\left|\left\langle n\left|\prod_{i} e^{i \mathbf{q}\left(x_{i}\right) \Delta x}\right| n_{0}\right\rangle\right|^{2}
$$

This is the main result in this paper. Notice that the factor $k_{n} / k_{n_{0}}$ does not appear in the WKB formula Eq. (20). In practice, one can evaluate Eq. (20) by diagonalizing $\mathbf{W}(x)$ at each $x_{i}$. This yields

$$
P=\sum_{n}\left|\left\langle n\left|\prod_{i}\left[\sum_{m}\left|m\left(x_{i}\right)\right\rangle e^{i q_{m}\left(x_{i}\right) \Delta x}\left\langle m\left(x_{i}\right)\right|\right]\right| n_{0}\right\rangle\right|^{2},
$$

where $|m(x)\rangle$ is the eigenvector of the matrix $\mathbf{W}(x)$ with the eigenvalue of $\lambda_{m}(x)$, and $q_{m}(x) \equiv \sqrt{2 m\left[E-\lambda_{m}(x)\right] / \hbar^{2}}$. For a single-channel problem, Eq. (20) is reduced to the familiar WKB formula, 


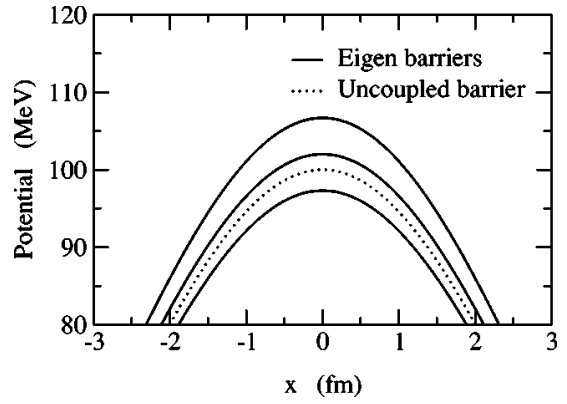

FIG. 1. The eigenpotentials for the three-channel problem obtained by diagonalizing the coupling matrix $\mathbf{W}(x)$ at each $x$ (the solid lines). The uncoupled barrier $V(x)$ is shown by the dotted line for a comparison.

$$
P(E)=\exp \left[-2 \int_{x_{0}}^{x_{1}} d x^{\prime} \sqrt{\frac{2 m}{\hbar^{2}}\left[V\left(x^{\prime}\right)-E\right]}\right],
$$

where $x_{0}$ and $x_{1}$ are the inner and the outer turning points, respectively.

Let us now apply the WKB formula (20) to a threechannel problem. We consider the following coupling potential:

$$
\mathbf{W}(x)=\left(\begin{array}{ccc}
V(x) & F(x) & 0 \\
F(x) & V(x)+\epsilon & F(x) \\
0 & F(x) & V(x)+2 \epsilon
\end{array}\right)
$$

with

$$
\begin{aligned}
& V(x)=V_{0} e^{-x^{2} / 2 s^{2}}, \\
& F(x)=F_{0} e^{-x^{2} / 2 s_{f}^{2}} .
\end{aligned}
$$

The parameters are chosen following Ref. [18] to be $V_{0}$ $=100 \mathrm{MeV}, F_{0}=3 \mathrm{MeV}$, and $s=s_{f}=3 \mathrm{fm}$, which mimic the fusion reaction between two ${ }^{58} \mathrm{Ni}$ nuclei. The excitation energy $\epsilon$ and the mass $m$ are taken to be $2 \mathrm{MeV}$ and $29 m_{N}$, respectively, where $m_{N}=938 \mathrm{MeV}$ is the nucleon mass. With these parameters, the three eigenbarriers $\lambda_{i}(x)$, which are obtained by diagonalizing $\mathbf{W}(x)$ at each $x$, have the barrier height of 97.31, 102.0, and 106.7 MeV, respectively, while the barrier height for the uncoupled barrier $V(x)$ is $100 \mathrm{MeV}$ (see Fig. 1).

Before we study the three-channel problem, let us first examine the validity of the WKB approximation for a singlechannel case to see whether the semiclassical approximation works in principle for the parameters that we choose. Figure 2 shows a comparison between the WKB penetrability for the uncoupled barrier $V(x)$ (the dashed line) obtained with Eq. (20) and the exact solution. It is plotted in linear and logarithmic scales in the upper and the lower panels, respectively. One clearly sees that the WKB approximation indeed works well at energies about $2 \mathrm{MeV}$ below the barrier height and lower.

As is well known, the naive WKB approximation breaks down around the barrier. In fact, the WKB penetrability is unity at the barrier height, while the exact result is about a

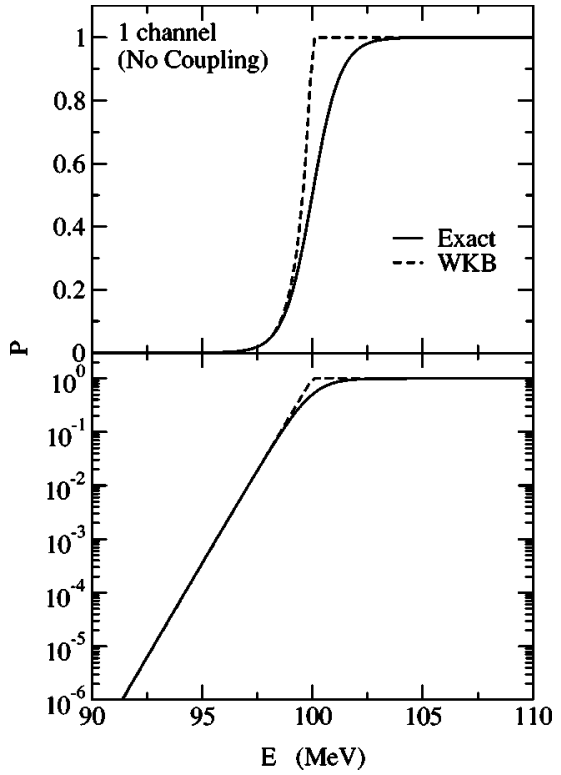

FIG. 2. The barrier penetrability for the uncoupled barrier $V(x)$ as a function of energy $E$ in linear (the upper panel) and the logarithmic (the lower panel) scales. The solid and the dashed lines are the exact solution and the WKB approximation, respectively.

half. Around these energies, one needs to improve the WKB formula by using the uniform approximation in order to take into account the multiple reflection of the classical path under the barrier $[19,20]$. We will discuss this problem more in the next section in connection to the penetrability in a coupled-channels system.

Let us now solve the coupled-channels problem, Eq. (23). We integrate Eq. (21) from $x=-15 \mathrm{fm}$ to $x=15 \mathrm{fm}$ with $\Delta x=0.05 \mathrm{fm}$. The dashed line in Fig. 3 shows the penetrability in the WKB approximation for this problem, which is compared to the exact solution of the coupled-channels equations (the solid line). The figure also shows the penetrability

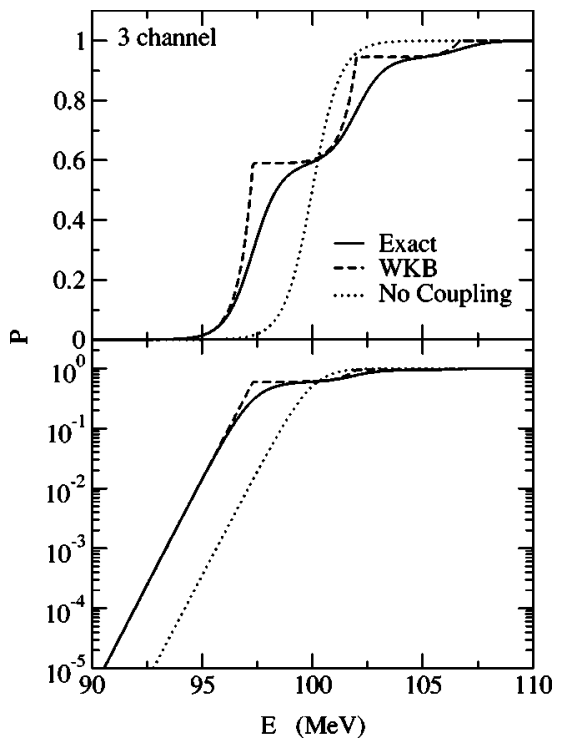

FIG. 3. Same as Fig. 2, but for the three-channel problem. The dotted line shows the penetrability in the no-coupling limit. 
for the uncoupled barrier as a comparison (the dotted line). Remarkably, the WKB formula (20) reproduces almost perfectly the exact solution at energies well below the lowest adiabatic barrier, i.e., $97.31 \mathrm{MeV}$, as in the single-channel problem.

We notice that the WKB penetrability increases much more rapidly than the exact penetrability at energies corresponding to the height of each eigenbarrier. This is in close analogy to the single-channel problem shown in Fig. 2. This behavior may be expected in the eigenchannel approach discussed in Refs. [18,21,22]. We will discuss this point in the next section.

\section{PENETRABILITY NEAR AND ABOVE THE BARRIER}

In the previous section, we showed that the multichannel WKB formula works remarkably well at energies well below the lowest eigenbarrier. Therefore, it can be expected that the WKB formula provides a useful framework in discussing, for instance, the role of inelastic excitations in the colliding nuclei in nuclear reactions at extremely low energies, such as astrophysically relevant reactions, where the standard coupled-channel calculations may be difficult to carry out.

At higher energies, however, we found that the agreement between the primitive WKB formula and the exact solution of the coupled-channel equations becomes poor. For a singlechannel problem, one can cure this problem by using the uniform approximation $[19,20]$. The WKB formula which is valid at all energies is given by

$$
P(E)=\frac{1}{1+\exp \left\{2 \int_{x_{0}}^{x_{1}} d x^{\prime} \sqrt{\left(2 m / \hbar^{2}\right)\left[V\left(x^{\prime}\right)-E\right]}\right\}},
$$

where the turning points $x_{0}$ and $x_{1}$ become complex numbers when the energy $E$ is above the barrier. It is not straightforward at all, however, to extend the uniform approximation to the coupled-channel problem. In this section, we instead present two prescriptions to deal with the coupled-channel penetrability at energies near and above the barrier.

\section{A. Dynamical norm method}

The first prescription is closely related to the dynamical norm method developed in Ref. [23]. It was argued in Ref. [23] that the penetrability may be expressed as a product of the penetrability in the adiabatic limit and a multiplicative factor to it which accounts for the nonadiabatic effect. The latter factor, which was called the dynamical norm factor, was evaluated through the imaginary time evolution for an intrinsic degree of freedom with a classical path obtained with the adiabatic potential.

We follow here the same idea as in the dynamical norm method, and reexpress Eq. (19) as

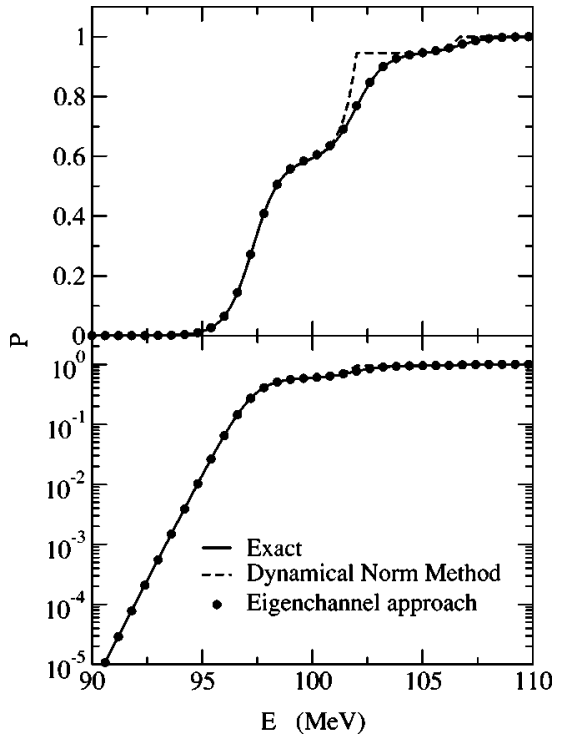

FIG. 4. Comparison of the barrier penetrability calculated by several methods. The solid line shows the exact solution of the coupled-channel equations. The dashed line is obtained with the modified adiabatic method which takes the nonadiabatic correction into account, while the filled circles are results of the eigenchannel approximation. The upper and the lower panels show the penetrability in linear and logarithmic scales, respectively.

$$
\boldsymbol{\tau}(-\infty)=\frac{1}{\sqrt{\mathbf{q}(-\infty)}}\left(\prod_{i} e^{i q_{0}\left(x_{i}\right) \Delta x} e^{i\left[\mathbf{q}\left(x_{i}\right)-q_{0}\left(x_{i}\right)\right] \Delta x}\right) \sqrt{\mathbf{q}(\infty)},
$$

where $q_{0}(x)=\sqrt{2 m\left[E-\lambda_{0}(x)\right] / \hbar^{2}}$ is the local wave number for the lowest eigenbarrier (i.e., the adiabatic barrier) $\lambda_{0}(x)$. The penetrability $P(E)$ is then given by

$$
\begin{aligned}
P= & \sum_{n}\left|\left\langle n\left|\prod_{i} e^{i\left[\mathbf{q}\left(x_{i}\right)-q_{0}\left(x_{i}\right)\right] \Delta x}\right| n_{0}\right\rangle\right|^{2} \\
& \times \exp \left[-2 \int_{x_{0}}^{x_{1}} d x^{\prime} \sqrt{\frac{2 m}{\hbar^{2}}\left[\lambda_{0}\left(x^{\prime}\right)-E\right]}\right] .
\end{aligned}
$$

The second factor on the right hand side (RHS) of this equation is nothing more than the WKB penetrability for the adiabatic potential $\lambda_{0}(x)$. At this stage, one may replace it by the exact penetrability for the adiabatic potential, $P_{\mathrm{ad}}(E)$. The first factor on the RHS of Eq. (28) expresses the nonadiabatic effect, as in the dynamical norm factor introduced in Ref. [23]. Notice that our formula, (28), is in fact an improvement of the dynamical norm method in Ref. [23], since the classical path is not assumed from the beginning in evaluating the dynamical norm factor.

The result of the modified dynamical norm method is shown in Fig. 4 by the dashed line. It is evident that the agreement between the WKB approximation and the exact solution improves significantly at energies near and slightly above the adiabatic barrier, although the method provides essentially the same result as the original WKB formula (20), at higher energies. 


\section{B. Eigenchannel approach}

The second prescription that we discuss is based on the eigenchannel approximation. In this approach, the penetrability is expressed as a weighted sum of the penetrability for the eigenbarriers $[18,21,22]$, that is,

$$
P(E)=\sum_{n} w_{n} P_{n}(E),
$$

where $P_{n}(E)$ is the penetrability for the eigenpotential $\lambda_{n}(x)$. The weight factors $w_{n}$ are usually estimated by assuming that the matrix $\mathbf{W}(x)$ is independent of $x$ through the interaction range $[18,22]$, while the coordinate dependence of $\mathbf{W}(x)$ is properly taken into account in calculating the penetrability $P_{n}(E)$. One often takes the barrier position of the uncoupled barrier, $X_{b}$, in order to estimate the weight factors [22]. This leads to [see Eq. (21)]

$$
w_{n}=w_{n}\left(X_{b}\right)=\left|\left\langle n\left(X_{b}\right) \mid n_{0}\right\rangle\right|^{2} .
$$

This procedure is indeed exact when the intrinsic degree of freedom has a degenerate spectrum [21,24-26], since the matrix $\mathbf{W}(x)$ can be diagonalized independent of $x$. When the intrinsic states have a finite excitation energy, however, the unitary matrix which diagonalizes $\mathbf{W}(x)$ explicitly depends on $x$, and the results may depend strongly on the position where the weight factors are evaluated. Also the weight factors possess some energy dependence in general. In Ref. [21], we have explicitly shown for a two-channel problem that the optimum weight factors are considerably different from those estimated at the barrier position, although their energy dependence appears to be weak. A satisfactory procedure to determine the weight factors has not been found so far when the excitation energy is finite.

In Fig. 3, we have shown that the WKB penetrability approaches a constant value at the barrier height of each eigenbarrier. Assuming that the weight factors are independent of energy, one can exploit this fact to determine a consistent value of the weight factors in the eigenchannel approach. For instance, at the barrier height of the lowest eigenbarrier, $E=B_{0}$, assuming that the contribution from the higher barriers is negligible, Eq. (29) suggests

$$
P\left(B_{0}\right) \sim w_{0} P_{0}\left(B_{0}\right) \sim w_{0},
$$

in the primitive WKB approximation (i.e., without the uniform approximation). Therefore, if one evaluates Eq. (20) at $E=B_{0}$, it directly provides the weight factor for the lowest eigenbarrier. One can repeat this procedure $N-1$ times to determine the weight factors $w_{0}, w_{1}, \ldots, w_{N-2}$ : suppose that the weight factors for the $k$ lowest eigenbarriers have been determined. The weight factor for the $(k+1)$ th eigenbarrier is then estimated as

$$
w_{k}=P_{\mathrm{WKB}}\left(B_{k}\right)-\sum_{i=0}^{k-1} w_{i},
$$

where $B_{k}$ is the barrier height of the $(k+1)$ th eigenbarrier, and $P_{\mathrm{WKB}}$ is the penetrability in the WKB approximation (20). Here, we have used the fact $P_{i}\left(B_{k}\right)=1$ for $i \leqslant k$ in the primitive WKB approximation, and assumed $P_{i}\left(B_{k}\right)=0$ for $i>k$. The weight factor for the highest eigenbarrier $\lambda_{N-1}(x)$ is evaluated as

$$
w_{N-1}=1-\sum_{i=0}^{N-2} w_{i}
$$

in order to ensure unitarity.

We apply this prescription to the three-channel problem discussed in the previous section. The weight factors are evaluated to be $0.5914,0.3543$, and 0.0543 for the lowest, the second lowest, and the highest eigenbarriers, respectively. The result of the eigenchannel approximation with the weight factors thus estimated is denoted by the filled circles in Fig. 4. The result is indistinguishable from the exact solution of the coupled-channel equations for all the energy region shown in the figure. We thus conclude that the multichannel WKB formula which we derive in this paper provides a consistent way to determine the weight factors in the eigenchannel approach, and it provides a useful and simple prescription to compute the penetrability in the presence of channel couplings at energies from well below to well above the potential barrier, as long as the weight factors are slowly varying functions of energy.

\section{SUMMARY}

We have extended the well-known WKB formula for barrier penetrability to systems with intrinsic degrees of freedom. Applying the formula to a three-channel problem, we have explicitly demonstrated that the WKB formula reproduces very nicely the exact solution of coupled-channel equations at energies well below the lowest eigenbarrier, i.e., the adiabatic barrier. The WKB formula which we derived is applicable even to systems with a large number of degrees of freedom, where the standard coupled-channel calculation cannot be performed due to a computational limitation. Our method may therefore provide a useful framework to discuss, e.g., a quantum scattering problem in the presence of coupling to a heat bath $[27,28]$. The WKB formula is also useful when one discusses the channel coupling effect on the tunneling rate at deep subbarrier energies, especially in the presence of closed channels, since the direct integration of the coupled-channel equations may suffer from a numerical instability. Such interesting problems include heavy-ion fusion reactions at extremely low energies [29], electron screening effects in nuclear astrophysical reactions [30,31], and nuclear structure effects in astrophysical fusion reactions [32,33].

The WKB formula that we derived neglects the effect of multiple reflection of a classical path under the potential barrier. Such primitive formula breaks down at energies near and above the adiabatic barrier, as is well known. We discussed two prescriptions to cure this problem. One is the dynamical norm method, where the WKB formula is reexpressed as a product of the penetrability for the adiabatic barrier and a multiplicative factor which accounts for the nonadiabatic effect. By replacing the adiabatic penetrability in the WKB approximation by the exact one, we have shown that this prescription improves the result at energies near and slightly above the adiabatic barrier. The second prescription 
is the eigenchannel approximation, where the penetrability is given as a weighted sum of the eigenpenetrability. By applying the WKB formula at energies corresponding to the barrier height of each eigenbarrier, we have presented a consistent procedure to determine the weight factors. We have shown that this prescription works well at energies from well below to well above the barrier, as long as the energy dependence of the weight factors is negligible. This method would provide a useful way to simplify the coupled-channel calculations in realistic systems.

For a single-channel problem, the primitive WKB formula can be improved by using the uniform approximation $[19,20]$, where the resultant WKB formula is applicable at energies both below and above the barrier. It will be an interesting, but very challenging, problem to extend it to a multichannel problem. For this purpose, it will be extremely helpful to construct a solvable coupled-channel model. Work in this direction is now in progress [34].

\section{ACKNOWLEDGMENTS}

The authors thank H. Horiuchi and Y. Fujiwara for useful discussions. A.B.B. gratefully acknowledges the 21st Century for Center of Excellence Program "Center for Diversity and Universality" at Kyoto University for financial support and thanks the Yukawa Institute for Theoretical Physics for its hospitality. This work was supported in part by the National Science Foundation, Grant No. PHY-0244384, and in part by the University of Wisconsin Research Committee with funds granted by the Wisconsin Alumni Research Foundation.
[1] A.B. Balantekin and N. Takigawa, Rev. Mod. Phys. 70, 77 (1998).

[2] M. Dasgupta, D.J. Hinde, N. Rowley, and A.M. Stefanini, Annu. Rev. Nucl. Part. Sci. 48, 401 (1998).

[3] K. Hagino, N. Rowley, and A.T. Kruppa, Comput. Phys. Commun. 123, 143 (1999).

[4] W. Brenig, T. Brunner, A. Gross, and R. Russ, Z. Phys. B: Condens. Matter 93, 91 (1993);W. Brenig and R. Russ, Surf. Sci. 315, 195 (1994).

[5] N. Yamanaka and Y. Kino, Phys. Rev. A 68, 052715 (2003).

[6] B.R. Johnson, J. Chem. Phys. 69, 4678 (1978).

[7] Z.H. Levine, Phys. Rev. A 30, 1120 (1984).

[8] T.N. Rescigno and A.E. Orel, Phys. Rev. A 25, 2402 (1982).

[9] K. Yabana and H. Horiuchi, Prog. Theor. Phys. 71, 1275 (1984).

[10] W. Van Dijk and M. Razavy, Can. J. Phys. 57, 1952 (1979).

[11] M. Razavy, Quantum Theory of Tunneling (World Scientific Publishing Co., Singapore, 2003).

[12] R. Bellman and R. Kalaba, Proc. Natl. Acad. Sci. U.S.A. 44317 (1958).

[13] H. Bremmer, Commun. Pure Appl. Math. 4, 105 (1951).

[14] R. Landauer, Phys. Rev. 82, 80 (1951).

[15] M.V. Berry, J. Phys. A 15, 3693 (1982).

[16] N.T. Maitra and E.J. Heller, Phys. Rev. A 54, 4763 (1996).

[17] S. Gasiorowicz, Quantum Physics (Wiley, New York 1974).

[18] C.H. Dasso, S. Landowne, and A. Winther, Nucl. Phys. A 405 381 (1983); 407, 221 (1983).

[19] D.M. Brink and U. Smilansky, Nucl. Phys. A 405, 301 (1983).
[20] D.M. Brink, Semi-Classical Methods for Nucleus-Nucleus Scattering (Cambridge University Press, Cambridge, England, 1985).

[21] K. Hagino, N. Takigawa, and A.B. Balantekin, Phys. Rev. C 56, 2104 (1997).

[22] C.H. Dasso and S. Landowne, Phys. Lett. B 183, 141 (1987).

[23] N. Takigawa, K. Hagino, and M. Abe, Phys. Rev. C 51, 187 (1995).

[24] K. Hagino, N. Takigawa, J.R. Bennett, and D.M. Brink, Phys. Rev. C 51, 3190 (1995).

[25] M.A. Nagarajan, A.B. Balantekin, and N. Takigawa, Phys. Rev. C 34, 894 (1986).

[26] H. Esbensen, Nucl. Phys. A 352, 147 (1981).

[27] B. Barkakaty and S. Adhikari, J. Chem. Phys. 118, 5302 (2003).

[28] A.H. Castro Neto and A.O. Caldeira, Phys. Lett. A 209, 290 (1995).

[29] K. Hagino, N. Rowley, and M. Dasgupta, Phys. Rev. C 67, 054603 (2003)

[30] F. Strieder, C. Rolfs, C. Spitaleri, and P. Corvisiero, Naturwissenschaften 88, 461 (2001).

[31] K. Langanke, in Advances in Nuclear Physics, edited by J.W. Negele and E. Vogt (Plenum, New York, 1993).

[32] K. Hagino, M.S. Hussein, and A.B. Balantekin, Phys. Rev. C 68, 048801 (2003).

[33] F.M. Nunes, R. Crespo, and I.J. Thompson, Nucl. Phys. A 615, 69 (1997).

[34] K. Hagino and A.B. Balantekin (unpublished). 